\title{
Effects of self-attraction and loading on annual variations of ocean bottom pressure
}

\author{
Nadya T. Vinogradova, ${ }^{1}$ Rui M. Ponte, ${ }^{1}$ Mark E. Tamisiea, ${ }^{2}$ James L. Davis, ${ }^{3}$ \\ and Emma M. Hill ${ }^{3}$ \\ Received 4 September 2009; revised 9 December 2009; accepted 24 February 2010; published 29 June 2010.
}

[1] The impact of self-attraction and loading (SAL) on ocean bottom pressure $\xi$, an effect not previously considered, is analyzed in terms of the mean annual cycle based on decadelong estimates of changes in land hydrology, atmospheric pressure, and oceanic circulation. The SAL-related changes in $\xi$ occur as a result of deformation of the crust due to loading and self-gravitation of the variable fluid loads. In the absence of SAL, net freshwater input and changes in mean atmospheric pressure over the ocean give rise to a spatially constant $\xi$ annual cycle with an amplitude $\sim 1-2 \mathrm{~cm}$ in equivalent water thickness. Consideration of SAL physics introduces spatial variations that can be significant, particularly around continental boundaries, where the amplitude of deviations can exceed $1 \mathrm{~cm}$. For the spatial variability induced by SAL effects, changes in both land hydrology and atmospheric pressure are important. Effects related to the changing ocean circulation are relatively weaker, apart from a few shallow coastal regions. Comparisons with a few in situ, deep ocean observations indicate that for the most accurate $\xi$ estimates, one needs to consider spatially varying SAL-related signals, along with the effects of mean atmospheric pressure and net freshwater input into the oceans. Nevertheless, the most complete estimates, including also effects of ocean circulation, are able to account for only $\sim 1 / 3$ of the observed annual variances. Sources of the remaining contribution remain unclear.

Citation: Vinogradova, N. T., R. M. Ponte, M. E. Tamisiea, J. L. Davis, and E. M. Hill (2010), Effects of self-attraction and loading on annual variations of ocean bottom pressure, J. Geophys. Res., 115, C06025, doi:10.1029/2009JC005783.

\section{Introduction}

[2] Ocean bottom pressure $(\xi)$ signals can be used to describe variability in ocean mass transports driven by surface wind stress and heat and freshwater exchanges with the atmosphere [e.g., Ponte, 1999]. Signals in $\xi$ can represent changes in deep ocean circulation, and thus be used to help infer changes in heat transports. Knowledge of $\xi$ is also needed to interpret fluctuations in sea level, which is an essential variable in ocean climate studies.

[3] Variability in $\xi$ at monthly and longer time scales has been studied using regional in situ observations [e.g., Hughes and Smithson, 1996], global gravity measurements from the GRACE (Gravity Recovery and Climate Experiment) mission [e.g., Ponte et al., 2007], and theoretical [e.g., Gill and Niiler, 1973] and numerical [e.g., Ponte, 1999] models. For the annual cycle of interest to us here, comparisons of model results and in situ observations have been

\footnotetext{
${ }^{1}$ Atmospheric and Environmental Research, Inc., Lexington, Massachusetts, USA.

${ }^{2}$ National Oceanography Centre, Liverpool, UK.

${ }^{3}$ Harvard-Smithsonian Center for Astrophysics, Cambridge, Massachusetts, USA.

Copyright 2010 by the American Geophysical Union. 0148-0227/10/2009JC005783
}

rare, but recent analyses of GRACE data reveal observed amplitudes considerably larger than the models indicate [Ponte et al., 2007]. Apart from possible problems with the observations, there might be some issues in the use of numerical models to simulate $\xi$ variability. For example, in order to account for non-Boussinesq processes, which are traditionally neglected in general circulation models, $\xi$ fields need to be corrected a posteriori to allow ocean mass rather than volume to be conserved [Ponte, 1999].

[4] Similarly ignored in numerical models are changes in global mean bottom pressure $\bar{\xi}$ (throughout the paper, overbar denotes quantities that are spatially averaged over the global oceans) that can be associated with net freshwater mass transfers into the ocean $\left(\bar{\xi}_{F}\right)$ and are related to the global hydrological cycle. Estimates of $\bar{\xi}_{F}$ have been made, however, using different methods, including temporal integration of global mean freshwater flux from atmospheric reanalysis [Vinogradov et al., 2008], using estimation from GRACE observations [Chambers et al., 2004], removing from altimetric sea level thermosteric effects calculated from in situ temperatures [Chen et al., 2005], and scaling the global mean halosteric height by an appropriate constant [Vinogradov et al., 2008]. These studies suggest a significant annual cycle in $\bar{\xi}_{F}$ with annual phase peaking around October and amplitude of $0.5-0.8 \mathrm{~cm}$. (Throughout the 
paper, values of $\xi$ are given in equivalent centimeters of water obtained by normalizing $\xi$ by constant gravity $g$ and constant seawater density $\rho_{0}$.) Another contribution to $\bar{\xi}$ that is usually not accounted for in numerical models is that associated with atmospheric pressure forcing. On seasonal scales, the resulting $\xi$ signal is spatially constant due to the ocean's isostatic response to atmospheric pressure loading [Ponte, 1999]. The amplitude of the annual cycle in the mean atmospheric pressure averaged over the global oceans $\left(\bar{\xi}_{A}\right)$ is significant and can reach up to $1 \mathrm{~cm}$ [Ponte et al., 2007].

[5] An effect that has not been considered at all in numerical studies of annual variability in $\xi$ is that of selfattraction and loading, abbreviated as SAL hereafter [after Gordeev et al., 1977]. A change in mass of the air and water column exerts a load on the seafloor, causing its deformation. The implicit change in earth and water mass distribution modifies the gravitational field, which leads to further adjustments of the mass field. These effects can act on the dynamical $\xi$ changes associated with atmospherically driven mass redistribution in the ocean. In addition, any change in mass loads over land from, e.g., hydrologic and cryospheric processes and changes in atmospheric pressure, leads to similar effects and can potentially affect $\xi$ fields.

[6] Analysis of SAL terms requires solving integrodifferential equations for $\xi$ using iterative methods or calculating convolution integrals on global grids [Stepanov and Hughes, 2004]. Such calculations are computationally expensive, which is part of the reason why these effects are not included in ocean circulation models. The importance of SAL for the study of tides has been long recognized. The effects are highly parameterized in tidal calculations and can change their amplitudes by up to 50\% [Ray, 1998; Gordeev et al., 1977]. The impact of SAL on the annual cycle in $\xi$ has never been studied, to our knowledge, but recently Tamisiea et al. [2010] have shown that SAL effects cause variations of the annual amplitude of relative sea level on the order of 1 to $2 \mathrm{~cm}$, supporting previous studies by Clarke et al. [2005], who found spatial patterns of sea level response being highly nonuniform. Such perturbations are similar in amplitude to expected $\xi$ variability at the annual period [Ponte, 1999; Vinogradov et al., 2008]. Here we attempt to evaluate the relative importance of the SAL and $\bar{\xi}$ effects on the annual cycle in $\xi$.

\section{Models}

[7] To investigate the magnitude of the SAL-related changes in $\xi\left(\xi_{S A L}\right)$ we use spatially varying monthly anomalies derived by Tamisiea et al. [2009] for the period from 1980 to 1997. A water load is introduced into the oceans that balances the change in water mass on the continents and in the atmosphere. An iterative solution of the spatial distribution of this water load is determined by assuming an initial uniform load together with the specified continental and atmospheric loads, calculating the impact on the crust and geoid height, and then allowing the ocean to adjust to these changes [Mitrovica and Peltier, 1991; Kendall et al., 2005]. The $\xi_{S A L}$ corrections are derived under the assumption that SAL effects give rise to a static ocean response. Three different effects are represented by (1) continental water mass changes using hydrological fields from LadWorld
[Milly and Shmakin, 2002], (2) atmospheric pressure using the reanalyses from the National Centers for Environmental Prediction/National Center for Atmospheric Research [Kalnay et al., 1996], and (3) dynamic bottom pressure driven by atmospheric forcing and provided by the output of the MIT general circulation model described by Hill et al. [2007]. The resulting $\xi$ changes due to these sources of mass variation are referred to as $\xi_{S A L_{H}}, \xi_{S A L_{A}}$, and $\xi_{S A L_{D}}$, respectively. Contributions of snow and ice from Greenland, Antarctica, Alaska and other sources to the total water storage are not considered here.Values of $\xi_{S A L}$ can be considered as a sum of its global mean and a deviation from that mean:

$$
\xi_{S A L}=\bar{\xi}_{S A L}+\Delta \xi_{S A L}
$$

The deviations $\Delta \xi_{S A L}$ represent spatially varying anomalies of the SAL-related changes in $\xi$. Computation of the global mean $\bar{\xi}_{S A L}$ term involves spatial integration of $\xi_{S A L}$ fields under different loads:

$$
\bar{\xi}_{S A L}=\frac{1}{A} \int_{A} \xi_{S A L} d A=\frac{1}{A} \int_{A}\left(\xi_{S A L_{H}}+\xi_{S A L_{A}}+\xi_{S A L_{D}}\right) d A
$$

where $A$ is the surface area of the global ocean. The first term in (2) represents net input of freshwater into the ocean from the land via the hydrological cycle; the second term represents freshwater exchange between the ocean and atmosphere due to changes in atmospheric water vapor; and the third term is equal to zero by definition. Thus, $\bar{\xi}_{S A L}$ reflects net mass transfer into the ocean due to freshwater flux:

$$
\bar{\xi}_{F}=\bar{\xi}_{S A L}
$$

[8] Time series of the global mean $\bar{\xi}$ is estimated as a sum of the net mass transfer into the ocean due to freshwater exchange and the mean surface atmospheric pressure over the ocean:

$$
\bar{\xi}=\bar{\xi}_{F}+\bar{\xi}_{A}
$$

where the atmospheric term $\bar{\xi}_{A}$ is calculated as by Ponte et al. [2007].

[9] The impact of the SAL and $\bar{\xi}$ terms is analyzed by comparing these signals with dynamic bottom pressure variations $\left(\xi_{d}\right)$ associated with mass redistribution in the ocean and generally driven by atmospheric surface forcing. Estimates of $\xi_{d}$ used here are based on monthly averaged fields from one of the ECCO solutions (version 2, iteration 216) described by Wunsch and Heimbach [2007] and Wunsch et al. [2009], which covers the period from 1992 to 2004. These solutions use the MIT general circulation model and its adjoint to produce an optimized estimate of the ocean state that is consistent with most available observations, each weighted according to the best existing estimate of the data and model errors. The optimization is achieved through adjustments of the initial temperature and salinity fields and the surface fluxes of momentum, heat and freshwater. To account for non-Boussinesq processes that are not represented in the solution, sea level and bottom pressure are corrected by unmodeled volume changes that are, following the approach described by Ponte [1999], equal to the spatial mean steric height. 
[10] As mentioned earlier, inserting the SAL effects directly into numerical ocean models involves calculating the SAL convolution integrals at each time step, which requires far more computing resources. Such procedures will be necessary when studying the ocean response at high frequencies, for which dynamic effects are likely to be important [Stepanov and Hughes, 2004]. In this study, however, we focus on monthly and longer scales at which an equilibrium response is assumed, and the effect of SAL on $\xi$ is calculated a posteriori after the model is run.

\section{Bottom Pressure Data}

[11] To investigate how well $\xi$ signals are modeled, and in particular to examine the relative importance of $S A L$ and $\bar{\xi}$ effects, it is useful to compare our $\xi$ estimates with observations. Satellite gravity data provide global $\xi$ fields but with considerable noise [Quinn and Ponte, 2008]. In this study, we choose instead to examine in situ deep ocean measurements from bottom pressure recorder (BPR) stations provided by several programs, including (1) DART (Deepocean Assessment and Reporting of Tsunamis) stations maintained by NOAA, (2) GLOUP (Global Undersea Pressure), and (3) ACCLAIM (Antarctic Circumpolar Current Levels by Altimetry and Island Measurements) maintained by the National Oceanography Centre, United Kingdom. Locations of BPR measurements can be seen in Figure 1.

[12] The DART BPRs collect data at a $15 \mathrm{~s}$ interval and record pressure in meters that represents the height of the ocean surface above the seafloor. The DART measurements are fairly recent and cover the period from 2003 to present. The GLOUP and ACCLAIM data contain pressure in millibars $(\mathrm{hPa})$ which is sampled either every $15 \mathrm{~min}$, hourly, or daily. The GLOUP measurements cover the period from 1975 to 1995 , and the ACCLAIM data contain the time period from 1985 to 2006, although there are many gaps during the sampling period. Prior to analysis, all data sets went through careful quality control, in which we estimated and eliminated artificial shifts in the data and averaged all data sets to daily series.

\section{Methods}

[13] The $\xi$ series are analyzed in terms of the mean annual cycle. The mean annual cycle is estimated by first averaging the fields corresponding to the months of January, February, etc. into a 12 month mean time series and then calculating the annual harmonic of this series. The mean annual cycle for the ECCO solution and SAL terms is computed based on 13 year and 17 year periods, respectively. The mean annual cycle for the in situ BPR observations is based on different record lengths depending on data availability. It is worth noting that the estimated mean annual cycle for the SAL and ECCO fields was not particularly sensitive to the time period as long as the series length was sufficiently long. Our sensitivity analyses, in which we compared annual cycles computed based on periods with different length, indicate that the root-mean-square (rms) difference between the mean annual cycle based on record lengths of 6 years and longer is only a few percent of the annual RMS values. Annual cycles of BPR observations, however, are usually based on a shorter period ( 1 to 3 years), and thus are less representative of true mean values.

[14] Prior to analysis, each data set was converted to monthly series that have had a mean removed. ECCO and SAL fields were defined on the same grid, and were collocated with BPR stations based on the grid point that is the nearest to the station location. Initially we started with 100 BPR stations that cover time periods longer than 1 year. A number of BPR stations contain $\xi$ measurements that were collected during different periods in time but at the same locations. Such stations, and also those stations that are located within $1^{\circ}$ of each other, are grouped together, which reduced the number of BPR series to 30 .

[15] In the comparisons with data, we examine three different models, gradually adding the effects of $\bar{\xi}$ and SAL to the fields $\xi_{d}$ from the ECCO estimates. The three models are defined as

$$
\begin{gathered}
m_{0}=\xi_{d} \\
m_{1}=\xi_{d}+\bar{\xi} \\
m_{2}=\xi_{d}+\bar{\xi}+\Delta \xi_{S A L}
\end{gathered}
$$

where model $m_{0}$ is the initial ECCO solution, model $m_{1}$ represents $\xi$ simulations corrected by the changes in temporally varying global mean bottom pressure $\bar{\xi}$, and $m_{2}$ is the most complete model that includes both temporal and spatial variations in $\xi_{S A L}$.

\section{Mean Annual Cycle of $\boldsymbol{\xi}$}

[16] As previously mentioned, the focus of this study is the mean annual cycle. Although high-frequency variations in $\xi$ can dominate the spectrum, annual signals in $\bar{\xi}$ and $\xi_{S A L}$ tend to be large compared to those at other periods, which would make these effects more significant. All bottom pressure results are presented in terms of equivalent water thickness.

\subsection{Annual Cycle in $\xi_{d}$ and $\bar{\xi}$}

[17] Amplitudes of the mean climatological annual cycle in $\xi_{d}$ estimated using the ECCO solution do not exceed $2 \mathrm{~cm}$, except for some areas in the Southern Ocean and along shallow coastal regions (Figure 1). In particular, in the Indian sector of the Southern Ocean $\xi_{d}$ shows strong annual variability with amplitudes comparable to those in sea level [e.g., Fukumori et al., 1998]. The phase of the mean annual cycle mostly displays a basin-scale pattern. Variations in $\xi_{d}$ over most of the Pacific and Indian oceans appear to be in phase, with the exception of the central part of the subtropical North Pacific gyre; seasonal changes in $\xi_{d}$ in the Atlantic and Pacific oceans are roughly out of phase with each other. The amplitude and phase patterns in Figure 1 are consistent with previous estimates by Ponte [1999] and Vinogradov et al. [2008].

[18] As previously mentioned, $\xi_{d}$ does not include the effects of $\bar{\xi}$ caused by the changes in atmospheric pressure $\left(\bar{\xi}_{A}\right)$ and those related to net freshwater exchange with the land and atmosphere $\left(\bar{\xi}_{F}\right)$, as defined in (4). Our estimates of $\bar{\xi}$ yield an annual cycle of about $2.5 \mathrm{~cm}$ peak to peak, with 

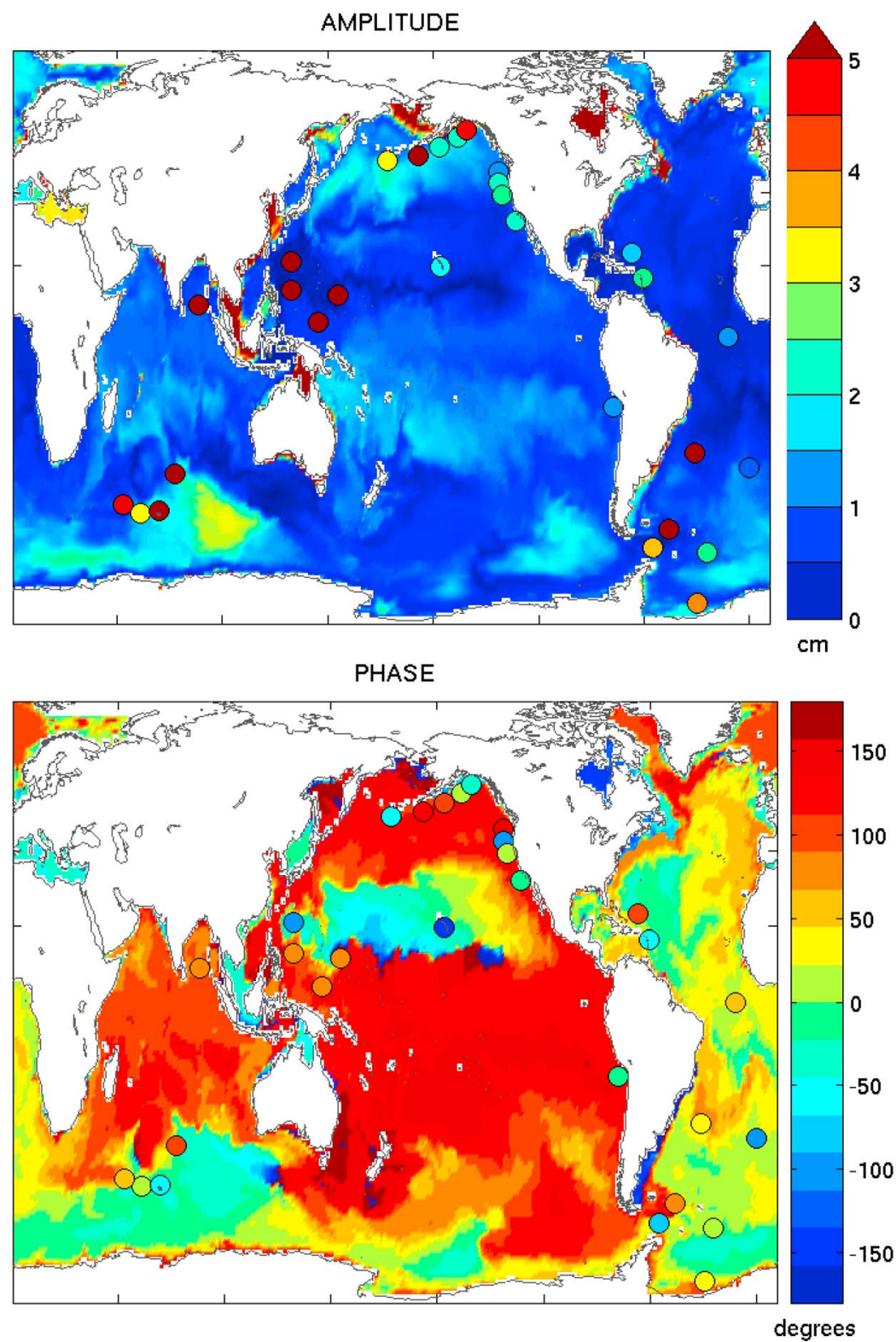

Figure 1. Amplitude and phase of mean annual cycle in $\xi_{d}$ estimates from the ECCO solution and estimates from in situ BPR measurements (shown as circles). All amplitude values are given in equivalent centimeters of water. The color scale extends beyond $5 \mathrm{~cm}$. Phase ranges from $-180^{\circ}$ to $+180^{\circ}$, with $-180^{\circ}$ phase corresponding to a peak occurring in mid-January, $-90^{\circ}$ corresponding to a peak in mid-April, $0^{\circ}$ corresponding to a peak in mid-July, $90^{\circ}$ corresponding to a peak in mid-October, and so on.

maximum value of $\bar{\xi}$ occurring in late August (Figure 2f). The atmospheric effect $\bar{\xi}_{A}$ has an annual amplitude of about $1 \mathrm{~cm}$, with a maximum in July, that is caused primarily by variable atmospheric air loadings, such as the shift of air masses toward Siberia in winter. Freshwater effects $\bar{\xi}_{F}$ amount to peak-to-peak annual variations of about $2 \mathrm{~cm}$, peaking in October. The estimates are consistent with those of Vinogradov et al. [2008], Ponte et al. [2007], and Chen et al. [2005], which are based on different methods, data types and time periods. Large amplitude annual cycles in $\bar{\xi}$, relative to $\xi_{d}$, indicate the significance of the annual oscillations in $\bar{\xi}$ and thus should be taken into account in studies of $\xi$.

\subsection{Annual Cycle in $\boldsymbol{\xi}_{S A L}$}

[19] Spatial patterns of the annual cycle in $\xi$ can be affected by $\xi_{S A L}$ fluctuations that are not treated in section 5.1. Figure 2 shows mean annual cycle of variations caused by different loads $\left(\xi_{S A L_{H}}, \xi_{S A L_{A}}\right.$, and $\left.\xi_{S A L_{D}}\right)$ and their combination $\left(\xi_{S A L}\right)$. As previously mentioned, the constant part of the SAL-related fluctuations $\bar{\xi}_{S A L}$ approximates net mass transfer into the ocean $\bar{\xi}_{F}$ due to freshwater flux from the land and atmosphere. The deviations from this mean $\left(\Delta \xi_{S A L}\right)$ result from the relative balance of the globally averaged background ocean mass variation and the local effects of the crustal deformation due to loading, the gravitational 

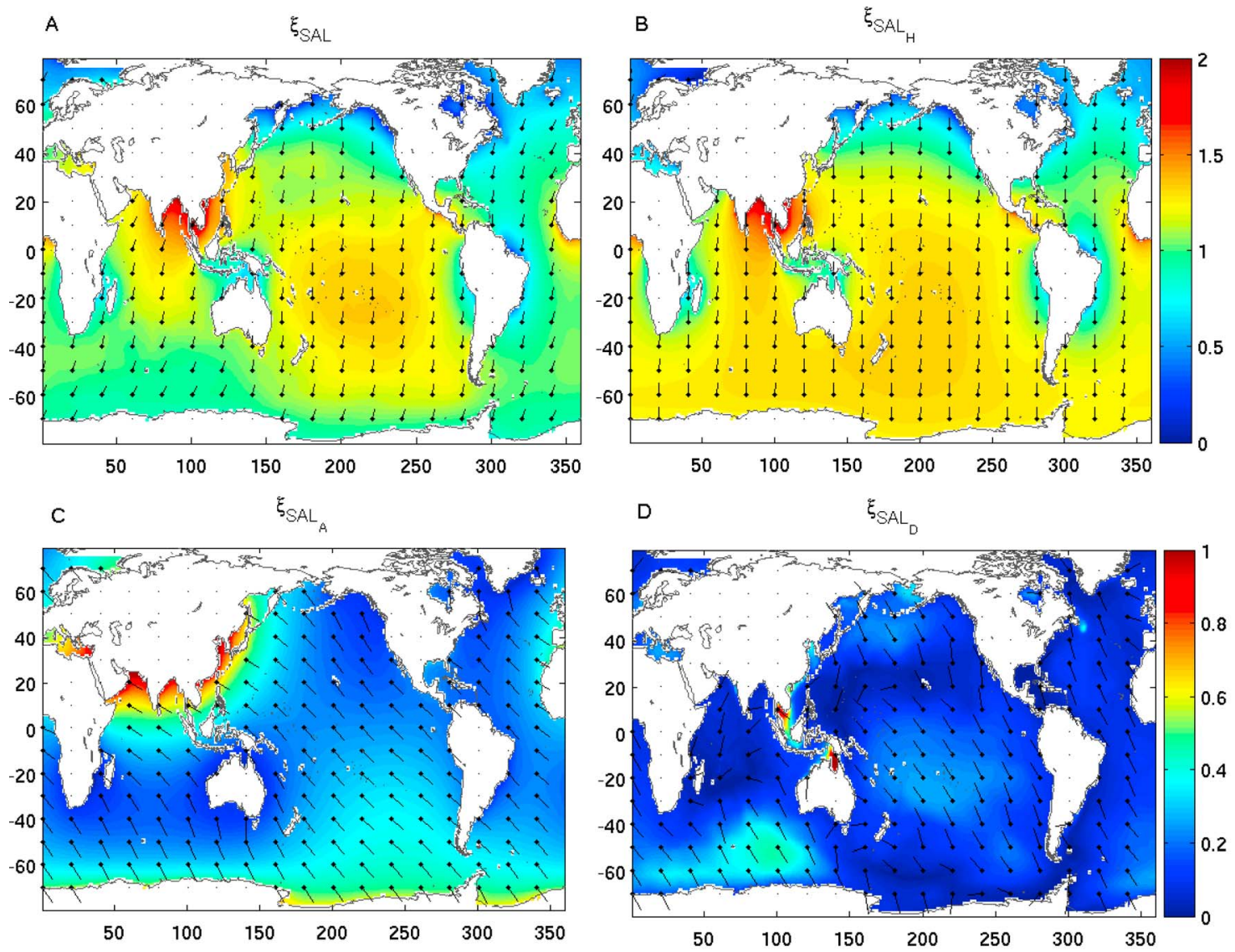

$\mathrm{E}$
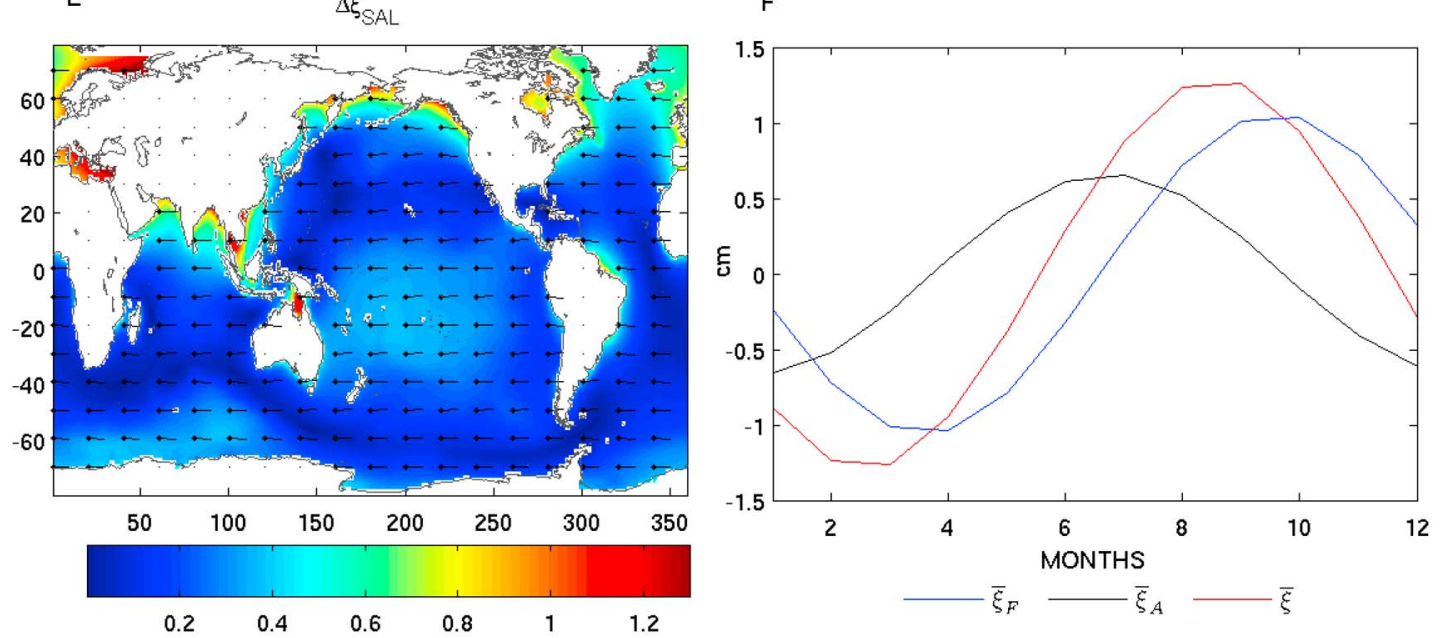

Figure 2. Annual amplitude (in $\mathrm{cm}$ ) and phase of $\xi$ corrections for the SAL effects under (b-d) individual loads and $(\mathrm{a}, \mathrm{e})$ their combination. Phases are indicated by the lines with the origin denoted by dots. A phase of $0^{\circ}$ corresponds to a peak in the month of July and is shown by a line pointing in the eastward direction; phase increases anticlockwise and is defined as in Figure 1. (f) Annual cycle of $\bar{\xi}$ is computed according to equation (4) as a sum of atmospheric $\left(\bar{\xi}_{A}\right)$ and freshwater $\left(\bar{\xi}_{F}\right)$ terms. The time labeling corresponds to the middle of the month from January to December.

effects of this deformation, and the direct gravitational attraction of the load. When a land coastal region loses a large volume of water, for example through glacial melt or river discharge, the local crust, including the nearby seafloor, uplifts and changes the local gravity field. In addition, because the mass in the region decreased, its gravitational attraction decreases, causing the nearby sea surface to fall. Simultaneous to these local changes, the total ocean mass is varying. These effects can amplify or cancel each other, resulting in spatial variability of the $\xi_{S A L}$ changes. 
[20] As seen in Figure 2, most of the deep ocean, including tropical regions, exhibit annual $\xi_{S A L}$ fluctuations of the order of $1-2 \mathrm{~cm}$ or smaller. Larger amplitudes $(>2 \mathrm{~cm})$ can be seen over parts of the Indian ocean. Among the three sources of mass variations considered in this study, hydrological load dominates $\xi_{S A L}$ (Figure 2b). Whereas most of the oceans are in phase, with the annual maximum occurring around October, the amplitude of $\xi_{S A L_{H}}$ varies in space, ranging from a few millimeters at high latitudes to about $2 \mathrm{~cm}$ in the Bay of Bengal. Spatial variability of $\xi_{S A L_{H}}$, with higher amplitudes in the Pacific, lower amplitudes in the Atlantic and variations along the coasts, reflects spatial distribution of hydrological processes. Estimates of the seasonal storage vary from less than $1 \mathrm{~cm}$ water equivalent in areas with uniform climatic conditions to $45 \mathrm{~cm}$ water equivalent in tropical river basins with a strong seasonal cycle [Riegger and Güntner, 2005].

[21] Variations in atmospheric pressure are the second largest contributor to the $\xi_{S A L}$ changes (Figure 2c). Spatial variations in annual amplitudes and phases of $\xi_{S A L_{A}}$ are significant, resulting from seasonal fluctuations in atmospheric pressure that cause a shift in air masses between the hemispheres and over different latitudinal zones. Although generally $\xi_{S A L_{A}}$ is weaker compared to $\xi_{S A L_{H}}$, they are of the same order of magnitude and thus $\xi_{S A L_{A}}$ needs to be taken into account. For example, in the regions around Antarctica and the Asian coastline, annual amplitudes of $\xi_{S A L}$ are large, responding to a strong seasonal cycle in atmospheric circulation in the southern polar cell and large seasonal variations of the Asian summer monsoon, respectively. In these regions, the amplitudes of $\xi_{S A L_{A}}$ and $\xi_{S A L_{H}}$ are comparable and their phase difference decreases the signal in the total $\xi_{S A L}$.

[22] Variations in $\xi_{S A L_{D}}$ are the weakest among the three loads (Figure 2d). However, there is a high level of spatial variability both in amplitude and phase. Variability of $\xi_{S A L_{D}}$ is higher in the Southern Ocean, Indonesian Seas, and in the Gulf of Carpentaria (northern Australia), where amplitudes of $1 \mathrm{~cm}$ are attained. Some regions of high $\xi_{S A L_{D}}$ variability correspond to closed contours of planetary potential vorticity $f / H$ (with $f$ being the Coriolis parameter and $H$ the ocean depth), and were pointed out as energetic regions by previous studies as well [e.g., Fukumori et al., 1998]. Overall, spatial patterns of $\xi_{S A L_{D}}$ are close to the amplitudes of $\xi_{d}$ seen in Figure 1. On average, the amplitude of $\xi_{S A L_{D}}$ represents about $15 \%$ of $\xi_{d}$ signal and can reach $20-30 \%$ in areas of high $\xi_{S A L_{D}}$ variability.

[23] The spatially varying patterns introduced by consideration of SAL physics and seen in $\Delta \xi_{S A L}$ (Figure 2e) illustrate most clearly the importance of the SAL effects over the globe. In the open ocean, $\xi_{S A L}$ and $\bar{\xi}_{S A L}$ are very similar, as indicated by the very small amplitude of $\Delta \xi_{S A L}$ $(0.2 \mathrm{~cm}$ or smaller aside from an area in the South Pacific). However, around many boundary regions, $\Delta \xi_{S A L}$ has an annual cycle with amplitudes of around $1 \mathrm{~cm}$ that are of the same order as $\xi$. Strong signals in $\Delta \xi_{S A L}$ are seen near Antarctica, the Gulf of Carpentaria, and the Amazon river basin, in response to individual loads of atmospheric pressure, dynamic ocean, and hydrology, respectively, or to their combination as, e.g., in Southeast Asia. In these regions, SAL effects play an important role in seasonal variations of $\xi$, and $\Delta \xi_{S A L}$ fluctuations cannot be neglected.

\subsection{Annual Cycle in Observations of Ocean Bottom Pressure}

[24] Observed amplitudes of the mean annual cycle in $\xi$, inferred from our BPR analysis, are shown as colored circles in Figure 1. The strongest annual amplitudes $(7-9 \mathrm{~cm})$ are detected in the Bay of Bengal, western Pacific and southern Aleutian Islands. Moderate annual amplitudes $(2-6 \mathrm{~cm}) \mathrm{can}$ be observed in the Indian Ocean sector of the Southern Ocean, southern Atlantic and in the Gulf of Alaska. The weakest annual amplitudes $(<2 \mathrm{~cm})$ are found along the western coast of the U.S., Hawaii and Central America. There are regions (e.g., in the Gulf of Alaska, along the western coast of the US) where observations show a high level of spatial variability in the annual cycle. In these regions, amplitudes between the nearest stations can differ by more than a factor of two, possibly reflecting sensitivity of $\xi$ to short length scale oceanic features, such as highly variable bottom topography in the area.

\section{Model and Data Comparison}

[25] To investigate the contribution of $\bar{\xi}$ and $\Delta \xi_{S A L}$ to the annual cycle of $\xi$ in a more quantitative manner, we compare $\xi$ measurements from the BPR stations with $\xi_{d}$ estimates corrected by the two effects defined in equations (5)-(7). The impact of each effect will be quantified in terms of the model/ data agreement.

[26] As mentioned earlier, comparisons of the simulated $\xi$ signals with in situ observations are rare. Hirose et al. [2001] compared high-frequency $\xi$ signals from a barotropic model with BPR observations at 18 sites in the Southern Ocean and showed that the simulated $\xi$ underestimated observed variances at all sites, with an average model/data correlation of 0.43 . Here we compare simulated and observed annual $\xi$ signals based on in situ measurements from 30 grouped BPRs. To quantitatively measure model/ data agreement we compare observations in terms of percentage of the annual variance explained by each model $m_{i}$ in (5)-(7). The explained variance is computed as

$$
\alpha_{i}=\frac{\sigma^{2}(d)-\sigma^{2}\left(d-m_{i}\right)}{\sigma^{2}(d)} \times 100 \%
$$

where $\sigma^{2}(X)$ denotes variance of $X, d$ are BPR observations, and $m_{i}$ for $i=0,1,2$ are models defined by (5)-(7). Values of the modeled and explained variances computed at $30 \mathrm{BPR}$ sites are given in Table 1 and Figure 3. With definition (8), high values of $\alpha$ indicate good model/data agreement.

[27] The ECCO solution $m_{0}$ explains a small percentage of annual variances, only $17 \%$ on average. Values of $\xi_{d}$ tend to underestimate observed variability almost at all sites (see standard deviations in Tables 1 and 2 for details). Typical values for the explained variance $\alpha_{0}$ are small $(<10 \%)$ or negative. A few exceptions of high $\alpha_{0}$ values include stations where both $\xi_{d}$ and data show strong signals (e.g., stations 4, 17).

[28] Adding a spatially constant correction $\bar{\xi}$ to the ECCO solution improves model/data agreement in half of the stations and almost doubles global average value of the explained variance. The number of stations where $m_{1}$ explains most of the data variance also increases (see Table 2). 

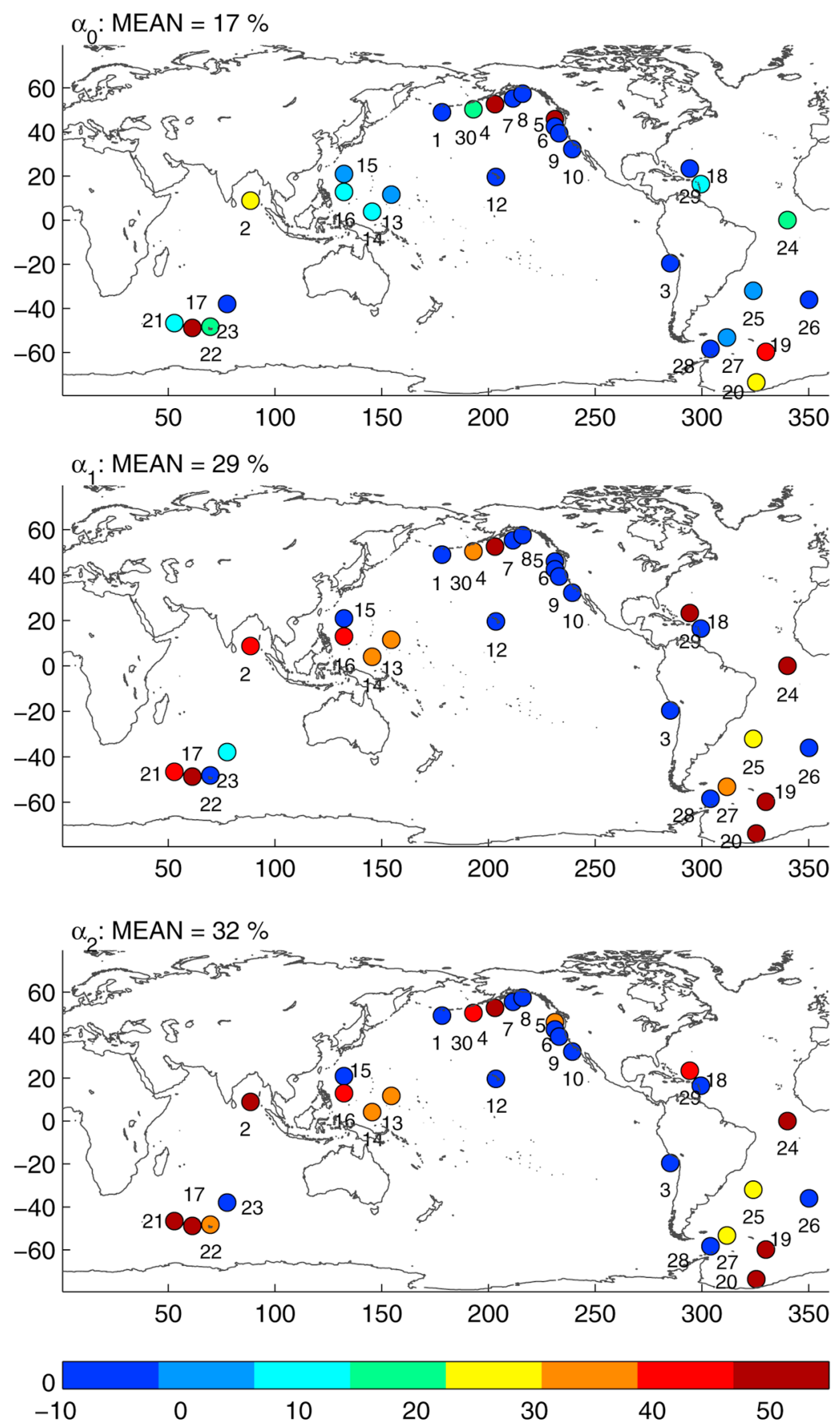

Figure 3. Percentage of annual variance explained by models (top) m_0, (middle) m_1, and (bottom) $\mathrm{m} \_2$, computed according to equation (8).

Typically, stations that exhibit smaller amplitudes in the data, i.e., $\leq 2-3 \mathrm{~cm}$, benefit the most due to the comparable amplitude of the $\bar{\xi}$ term (e.g., station 24 in Figure 4). However, if there is a phase shift between $m_{0}$ and observed annual cycles, $m_{1}$ does not improve model/data agreement. See for example stations along the North American west coast (stations 5 and 10 in Figure 4). As was discussed in section 5.3, these regions show high spatial variability, with quite different annual cycles at stations that are geographically close, which indicates the importance of fine spatial structures that are not resolved by coarse-resolution model $m_{0}$.

[29] Spatially varying correction $\Delta \xi_{S A L}$ can further improve model/data agreement, although the difference between $m_{2}$ and $m_{1}$ is less dramatic compared to that between $m_{1}$ and $m_{0}$. On average, $m_{2}$ improves the variance explained by $\sim 3 \%$ mostly by reducing the number of negative values of $\alpha_{2}$ compared to $\alpha_{1}$. Typically, $m_{2}$ performs better at stations where $\Delta \xi_{S A L}$ amplitudes are large (e.g., Bay of Bengal, station 2 in Figure 4) and phase representation is better 
Table 1. Annual Variance Explained by Each Model, Standard Deviation of the $\xi_{d}$ Annual Amplitudes From the ECCO 13 Year Series, and Standard Deviation of the Data and Each Model

\begin{tabular}{|c|c|c|c|c|c|c|c|c|}
\hline Station & $\begin{array}{c}\alpha_{0} \\
(\%)\end{array}$ & $\begin{array}{c}\alpha_{1} \\
(\%)\end{array}$ & $\begin{array}{c}\alpha_{2} \\
(\%)\end{array}$ & $\mathrm{ERR}^{\mathrm{a}}$ & $\begin{array}{l}\sigma(D) \\
(\mathrm{cm})\end{array}$ & $\begin{array}{c}\sigma\left(m_{0}\right) \\
(\mathrm{cm})\end{array}$ & $\begin{array}{c}\sigma\left(m_{1}\right) \\
(\mathrm{cm})\end{array}$ & $\begin{array}{c}\sigma\left(m_{2}\right) \\
(\mathrm{cm})\end{array}$ \\
\hline 1 & -14 & -24 & -23 & 0.4 & 9.4 & 1.7 & 2.2 & 2.2 \\
\hline 2 & 24 & 45 & 52 & 0.2 & 7.6 & 1.2 & 1.9 & 2.1 \\
\hline 3 & -19 & -40 & -34 & 0.2 & 3.8 & 1.1 & 1.6 & 1.5 \\
\hline 4 & 81 & 98 & 97 & 0.5 & 3.9 & 1.6 & 2.1 & 2.0 \\
\hline 5 & 91 & -4 & 37 & 0.3 & 2.0 & 1.0 & 1.5 & 1.2 \\
\hline 6 & -8 & -24 & -19 & 0.4 & 4.3 & 1.0 & 1.5 & 1.4 \\
\hline 7 & -7 & -10 & -9 & 0.4 & 2.4 & 1.3 & 1.9 & 1.7 \\
\hline 8 & -5 & -8 & -7 & 0.3 & 4.9 & 1.2 & 1.7 & 1.5 \\
\hline 9 & -3 & -5 & -4 & 0.4 & 3.5 & 1.1 & 1.6 & 1.5 \\
\hline 10 & -8 & -14 & -13 & 0.4 & 1.9 & 1.1 & 1.6 & 1.6 \\
\hline 11 & 74 & 91 & 95 & 0.7 & 3.6 & 2.8 & 3.0 & 3.0 \\
\hline 12 & -1 & -15 & -16 & 0.4 & 6.2 & 1.0 & 1.2 & 1.3 \\
\hline 13 & 2 & 32 & 35 & 0.4 & 5.9 & 1.0 & 1.4 & 1.5 \\
\hline 14 & 9 & 31 & 32 & 0.4 & 9.4 & 1.1 & 1.6 & 1.6 \\
\hline 15 & 2 & -3 & -3 & 0.4 & 10.3 & 1.1 & 1.4 & 1.5 \\
\hline 16 & 10 & 39 & 42 & 0.4 & 6.1 & 1.1 & 1.6 & 1.7 \\
\hline 17 & 74 & 91 & 95 & 0.7 & 3.6 & 2.8 & 3.0 & 3.0 \\
\hline 18 & 8 & -7 & -6 & 0.5 & 3.7 & 0.7 & 1.1 & 1.0 \\
\hline 19 & 46 & 76 & 80 & 0.7 & 2.7 & 2.1 & 2.3 & 2.3 \\
\hline 20 & 24 & 59 & 59 & 0.7 & 3.7 & 2.3 & 2.6 & 2.6 \\
\hline 21 & 9 & 44 & 50 & 0.3 & 4.3 & 0.8 & 1.3 & 1.3 \\
\hline 22 & 16 & 0 & 34 & 0.5 & 8.4 & 1.1 & 1.3 & 1.4 \\
\hline 23 & -2 & 7 & 2 & 0.3 & 8.4 & 0.9 & 1.2 & 1.3 \\
\hline 24 & 22 & 100 & 98 & 0.2 & 2.2 & 0.4 & 1.0 & 1.0 \\
\hline 25 & 6 & 26 & 23 & 0.4 & 7.9 & 0.8 & 1.4 & 1.3 \\
\hline 26 & -6 & -58 & -58 & 0.3 & 2.7 & 0.8 & 1.2 & 1.2 \\
\hline 27 & 1 & 31 & 30 & 0.5 & 5.8 & 0.8 & 1.2 & 1.2 \\
\hline 28 & -0 & -7 & -7 & 0.0 & 9.8 & 0.1 & 0.8 & 0.8 \\
\hline 29 & -2 & 55 & 50 & 0.3 & 2.0 & 0.4 & 0.9 & 0.8 \\
\hline 30 & 21 & 35 & 43 & 0.3 & 7.2 & 1.1 & 1.7 & 1.6 \\
\hline
\end{tabular}

${ }^{\mathrm{a}}$ ERR, standard deviation of the $\xi_{d}$ annual amplitudes from the ECCO 13 year series.

(e.g., station 22 in Figure 4). This result indicates that spatial variability provided by $\xi_{S A L}$ effects is important in the treatment and understanding of $\xi$ seasonal signals.

[30] Simulated and observed seasonal variations in $\xi$ are compared in terms of the mean annual cycle, which is based on a 13 year period for the models and 1-3 year period for the data, depending on availability of observations. To assess the uncertainty related to averaging over different time periods, we compute the standard deviation of $\xi_{d}$ annual amplitudes during the 13 year period. The values shown in Table 1 range from a few millimeters to less than $1 \mathrm{~cm}$, with a global average value of $0.3 \mathrm{~cm}$, which is only about $10 \%$ of the global average amplitude of the BPR mean annual cycle. In contrast, a number of amplitudes of BPR and the various models differ by factors of more than two. Thus, most of these differences cannot be attributed to the expected year-to-year variability of the annual cycle and the different record lengths used in our comparisons.

\section{Conclusions}

[31] Annual variability in $\xi$ reflects in part the internal mass redistribution associated with the ocean dynamic response to momentum, heat and freshwater fluxes at the boundaries with the atmosphere and land. The wind stress curl is particularly significant in driving large-scale dynamic signals $\xi_{d}$ [Ponte, 1999; Gill and Niiler, 1973]. Fluctuations in $\xi_{d}$ are commonly treated in model-based estimates of $\xi$ and, apart from shallow coastal regions, have annual amplitudes of order $1 \mathrm{~cm}$ (Figure 1). Two other effects on $\xi$ arise from net freshwater exchanges with the land and atmosphere and from changes in surface atmospheric pressure over the oceans. Under a quasi-static response to loading, such effects give rise to spatially constant annual signals in $\xi$ [Ponte, 1999]. These signals are usually not represented in ocean models, as they are not dynamically relevant, but we find that they have annual amplitudes comparable to $\xi_{d}$ (Figure 2) and thus are very important to consider when comparing and combining models and observations of the annual cycle in $\xi$, as also found in previous studies [e.g., Ponte et al., 2007].

[32] A component of the $\xi$ signal that has not been considered before has to do with the physics of SAL. Annual fluctuations in $\xi$ associated with SAL effects were documented here (Figure 2) based on the calculations of Tamisiea et al. [2010]. The spatial variations $\Delta \xi_{S A L}$ associated with SAL effects (Figure 2e) can introduce changes in $\xi$ annual amplitudes ranging from a few millimeters to more than $1 \mathrm{~cm}$, which are of the same order of magnitude of the seasonal variations in $\xi_{d}$ over many ocean regions. The importance of spatially varying $\xi_{S A L}$ corrections is particularly clear in coastal regions, where the amplitude of $\Delta \xi_{S A L}$ anomalies can reach more than $1 \mathrm{~cm}$.

[33] Spatial patterns in $\xi_{S A L}$ fields are primarily driven by seasonal changes in land hydrology, which yield strongest amplitudes near India and Southeast Asia and weakest amplitudes in the high northern latitudes (Figure 2b). Seasonal changes in atmospheric pressure drive significant spatial anomalies around Asia and Antarctica, and in the Mediterranean (Figure 2c). Effects of SAL associated with the dynamic component $\xi_{d}$ are generally weaker, except in some shallow coastal regions where strong annual variability is found. Our results point to the need to account for all these SAL effects when treating the annual cycle in $\xi$.

[34] The ability to represent and understand the variability of $\xi$ seen in the records, and in particular the annual cycle, continues to be a challenge. Our current "best" estimates, combining $\xi_{d}$, the spatially varying $\xi_{S A L}$ and constant $\bar{\xi}$ corrections, can explain on average $32 \%$ of the variance in the observed annual cycle in the set of BPRs analyzed here. This value is significantly higher than the value of $17 \%$ obtained when only $\xi_{d}$ estimates are used. However, even the most complete $\xi$ estimates considered here fall short of explaining the observed annual variability. Similar findings result from comparisons with GRACE data [Ponte et al., 2007].

[35] Apart from uncertainties in the measurements, including the limited temporal coverage of BPRs that limit the ability to calculate a robust mean annual cycle, a long

Table 2. Statistical Summary of Table 1

\begin{tabular}{ccccc}
\hline Model & $\begin{array}{c}\text { Global Average } \\
\text { Percent }\end{array}$ & $\begin{array}{c}\text { Percent Where } \\
\alpha<10 \%\end{array}$ & $\begin{array}{c}\text { Percent Where } \\
\alpha>40 \%\end{array}$ & $\begin{array}{c}\text { Percent Where } \\
\alpha \geq 80 \%\end{array}$ \\
\hline$m_{0}$ & 17 & 63 & 17 & 10 \\
$m_{1}$ & 29 & 50 & 30 & 13 \\
$m_{2}$ & 32 & 43 & 36 & 17 \\
\hline
\end{tabular}



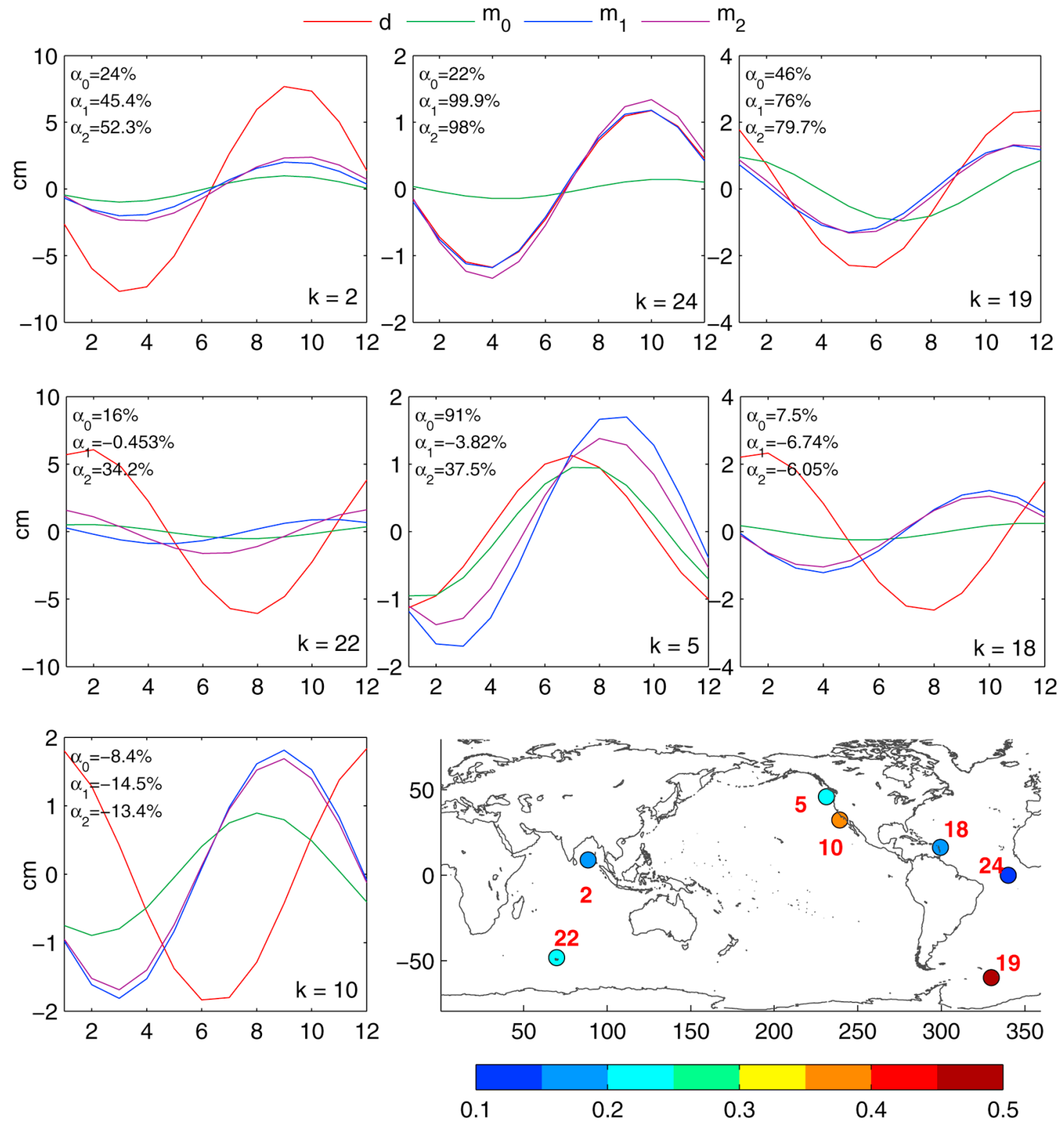

Figure 4. Mean annual cycles of the data $(D)$ and the initial $\left(m_{0}\right)$ and corrected ECCO solutions $\left(m_{1}\right.$ and $m_{2}$ ) at selected locations. Station locations and uncertainties of the estimated annual cycle (in $\mathrm{cm}$ ) are shown as colored circles.

list of errors affecting our estimates of $\xi$ is possible [e.g., Ponte et al., 2007]. Insufficient knowledge of the atmosphere and hydrology fields driving $\xi_{d}$ and $\xi_{S A L}$ is always an issue, but perhaps most important for the case of BPR data comparisons is the use of smoothed topography and coarse spatial resolution $\left(1^{\circ}\right)$ in estimating $\xi_{d}$. The BPR data represent point measurements that can be affected by fine structures in $\xi_{d}$ variability, possibly related to topography. Such high spatial variability will be absent from model estimates of $\xi_{d}$ and can perhaps explain some of the model/data discrepancies discussed in this paper.

[36] One way to mitigate the errors in $\xi$ estimates is to assimilate observations of $\xi$ into ocean models. Such undertakings, currently being considered for both BPR and GRACE data, require estimates of the data uncertainties, and the model/data comparisons presented here provide a good basis to arrive at proper weights for BPR data, necessary for the constraining procedures. Another way for error mitigation is to include missing physics in numerical ocean models as much as possible. While seasonal changes in $\xi_{S A L}$ have been used here under the assumption that SAL effects give rise to a static response, the equilibrium assumption most likely does not hold at higher frequencies [Stepanov and Hughes, 2004], in which case explicit inclusion of SAL physics in time-stepping numerical ocean models will be necessary.

[37] Acknowledgments. The authors are grateful to Patrick Heimbach for making ECCO model output available. The work was supported in part by NASA grants NNX07AM77G and NNX08AJ79G. Additional support was provided by the National Oceanographic Partnership Program through its sponsorship of the ECCO project and by the Natural Environment 
Research Council's Oceans 2025 Program. Useful interactions in the course of this work with many ECCO colleagues are gratefully acknowledged. The authors also thank two anonymous reviewers for commenting on the manuscript.

\section{References}

Chambers, D. P., J. Wahr, and R. S. Nerem (2004), Preliminary observations of global ocean mass variations with GRACE, Geophys. Res. Lett., 31, L13310, doi:10.1029/2004GL020461.

Chen, J. L., C. R. Wilson, B. D. Tapley, J. S. Famiglietti, and M. Rodell (2005), Seasonal global mean sea level change from satellite altimeter, GRACE, and geophysical models, J. Geod., 79, 532-539.

Clarke, P. J., D. A. Lavallée, G. Blewitt, T. M. van Dam, and J. M. Wahr (2005), Effect of gravitational consistency and mass conservation on seasonal surface mass loading models, Geophys. Res. Lett., 32, L08306, doi:10.1029/2005GL022441.

Fukumori, I., R. Raghunath, and L.-L. Fu (1998), Nature of global largescale sea level variability in relation to atmospheric forcing: A modeling study, J. Geophys. Res., 103, 5493-5512.

Gill, A. E., and P. P. Niiler (1973), The theory of the seasonal variability in the ocean, Deep Sea Res. Oceanogr. Abstr., 20, 141-177.

Gordeev, R. G., B. A. Kagan, and E. V. Polyakov (1977), The effects of loading and self-attraction on global ocean tides: The model and the results of a numerical experiment, J. Phys. Oceanogr., 7, 161-170.

Hill, E. M., R. M. Ponte, and J. L. Davis (2007), Dynamic and regression modeling of ocean variability in the tide-gauge record at seasonal and longer periods, J. Geophys. Res., 112, C05007, doi:10.1029/ 2006JC003745.

Hirose, N., I. Fukumori, V. Zlotnicki, and R. M. Ponte (2001), Modeling the high-frequency barotropic response of the ocean to atmospheric disturbances: Sensitivies to forcing, topography, and friction, J. Geophys. Res., 106, 30,987-30,995.

Hughes, C. W., and M. J. Smithson (1996), Bottom pressure correlations in the South Atlantic, Geophys. Res. Lett., 23, 2243-2246.

Kalnay, E., et al. (1996), The NCEP/NCAR 40-year reanalysis project, Bull. Am. Meteorol. Soc., 77, 437-470.

Kendall, R. A., J. X. Mitrovica, and G. A. Milne (2005), On post-glacial sea level-II. Numerical formulation and comparative results on spherically symmetric models, Geophys. J. Int., 161, 679-706, doi:10.1111/ j.1365-246X.2005.0553.x.

Milly, P. C., and A. B. Shmakin (2002), Global modeling of land water and energy balances. Part I: The land dynamics (LaD) model, J. Hydrometeorol., 3, 283-299.
Mitrovica, J. X., and W. R. Peltier (1991), On postglacial geoid subsidence over the equatorial oceans, J. Geophys. Res., 96, 20,053-20,071.

Ponte, R. M. (1999), A preliminary model study of the large-scale seasonal cycle in bottom pressure over the global ocean, J. Geophys. Res., 104, 1289-1300.

Ponte, R. M., K. J. Quinn, C. Wunsch, and P. Heimbach (2007), A comparison of model and GRACE estimates of the large-scale seasonal cycle in ocean bottom pressure, Geophys. Res. Lett., 34, L09603, doi:10.1029/ 2007GL029599.

Quinn, K. J., and R. M. Ponte (2008), Estimating weights for the use of time-dependent gravity recovery and climate experiment data in constraining ocean models, J. Geophys. Res., 113, C12013, doi:10.1029/ 2008JC004903.

Ray, R. D. (1998), Ocean self-attraction and loading in numerical tidal models, Mar. Geod., 21, 181-192.

Riegger, J., and A. Güntner (2005), Time variation in hydrology and gravity, Earth Moon Planets, 94, 41-55, doi:10.1007/s11038-005-1831-8.

Stepanov, V. N., and C. W. Hughes (2004), Parameterization of ocean selfattraction and loading in numerical models of the ocean circulation, J. Geophys. Res., 109, C03037, doi:10.1029/2003JC002034.

Tamisiea, M. E., E. M. Hill, R. M. Ponte, J. L. Davis, I. Velicogna, and N. T. Vinogradova (2010), Impact of self-attraction and loading on the annual cycle in sea level, J. Geophys. Res., doi:10.1029/2009JC005687, in press. Vinogradov, S. V., R. M. Ponte, P. Heimbach, and C. Wunsch (2008), The mean seasonal cycle in sea level estimated from a data-constrained general circulation model, J. Geophys. Res., 113, C03032, doi:10.1029/ 2007JC004496.

Wunsch, C., and P. Heimbach (2007), Practical global oceanic state estimation, Physica D, 230, 197-208.

Wunsch, C., P. Heimbach, R. M. Ponte, I. Fukumori, and ECCO-GODAE Consortium Members (2009), The global general circulation of the oceans estimated by the ECCO-consortium, Oceanography, 22, 88-103.

J. L. Davis and E. M. Hill, Harvard-Smithsonian Center for Astrophysics, 60 Garden St., Cambridge, MA 02138, USA.

R. M. Ponte and N. T. Vinogradova, Atmospheric and Environmental Research, Inc., 131 Harwell Ave., Lexington, MA 02421, USA. (nadya@aer.com)

M. E. Tamisiea, National Oceanography Centre, 6 Bronwlow St., Liverpool L3 5DA, UK. 